\title{
DISCOVERING ADAPTIVE WIRELESS SENSOR NETWORK USING B-SYNCHRONIZER
}

\author{
Pradhnya S. Kalaskar ${ }^{1}$, Prachi Jaini ${ }^{2}$ \\ ${ }^{I}$ Department of Computer Science \& Engineering, G. H. Raisoni College of Engineering, Nagpur-16, India \\ ${ }^{2}$ Department of Computer Science \& Engineering, G. H. Raisoni College of Engineering, Nagpur-16, India
}

\begin{abstract}
When we consider the standard Bellman-Ford algorithm, it uses static values of link cost function and distance function. These static values are stored in sink node so that the sink node requires memory to keep data safe. Therefore the space, message and time complexity of a network and node increases. To overcome this we discover Fast Time Dependent Shortest Path algorithm with message and used in network with $\beta$ synchronizer. The FTSP algorithm uses dynamic values of link cost function and distance function and to store these values we are using vector compression method so that there is no need to store the data into the sink node. Because of this the message, time and space complexity of node will be decreases.
\end{abstract}

Keywords- Duty cycle, Time dependent, $\beta$ synchronizer

\section{INTRODUCTION}

When we consider the traditional MAC protocols which are used to maximize the packet throughput, provide fairness, and minimize latency. But it consumes more power in wireless sensor network where the energy is an important constraint. To reduce the energy consumption of a node there is strong requirement of reducing the latency of a node, reducing the average time required o complete one duty cycle and total message count which are used to update the node information.

There are various sources which are used in wireless sensor network but it consumes huge amount of energy for example overhearing, collision of packets, idle listening and control packet overhead [10]. Because of this the valuable data will be affected from the network while communication is going on.

The budget of wireless sensor network is very limited so that it is not possible to maintain the communication with central coordinator. This type of network topology is partitioned and time-dependent. The time factor is most important in wireless sensor network hence synchronization between nodes in the network should be strong.

For designing the adaptive network the shortest path algorithm should be strong so that it will help to reduce the power consumption of a node, message, space and time complexity of a sink node. In wireless sensor network the sink node is responsible for whole communication from source to destination. Sink node stores the all necessary information of a node like IP-address of source and destination, link updates, network update. To store such type of information the sink node requires the enough memory.
In our proposed FTSP algorithm there are two stages for constructing shortest path, first stage is building spanning tree and in the second stage calculating the shortest path and sends an acknowledgement to the sink node.

The FTSP algorithm works with the $\beta$ synchronizer. $\beta$ synchronizer is a special type of synchronizer used to broadcast the packets in the network and to decrease the message complexity of asynchronous systems [1]. The $\beta$ synchronizer has an initialization phase in which nodes are initialized in the network, from which one leader node is selected and the spanning tree is rooted around this leader. After execution of one phase the leader node learns that all the nodes in the network are "safe" and then broadcasts the message along the spanning tree. All the nodes in the network then generate new clock pulse. After that $\beta$ synchronizer learns that all the nodes in the network are safe or not once it is cleared then it sends an acknowledgement to the parent node [1].

\section{RELATED WORK}

The time dependent shortest path algorithm was first studied for GPS navigation and for centralized approach. It defines the two different policies for time dependent shortest path one is waiting policy and second is non waiting policy. Waiting means wait for data to be delivered to the destination. The non waiting means the sender sends the packet over network when the receiver awake [1].

Low power listening was used by CSMA based technology to achieve the low power communication. The B-MAC uses the independent sleep and awake mechanism for scheduling. But BMAC consumes the energy and it also surpasses the existing latency and throughput. 
To overcome this disadvantage the XMAC and DSP-MAC was proposed with short preamble for replacing the long preamble in B-MAC. To overcome the overhearing problem, the receiver's information was also embedded in short preamble [1].

Based on the node's residual energy adaptive low power listening was proposed in [15]. ALPL supports point-to-point latency and time dependent link cost.

Delay is also an important factor for routing in wireless sensor network. Routing becomes too difficult in wireless sensor network because of two reasons one by changing in transmission latency and other one is intermittent connection between two neighbour nodes.

To overcome this disadvantage the on demand routing approach was proposed which were used the probe messages for determining the least latency route.

\section{PROPOSED WORK}

\subsection{Maintaining the Dynamic Route}

When we consider the static network the sink node require to store the route information so that when the link changes or node changes its duty cycle or if it also joins any dynamic network, the link which is connecting to all nodes in the network will be changed multiple times. Therefore the sink node will be overloaded and multiple links will be affected.

To maintain the dynamic route of a multicast network our proposed algorithm stores only the route information in the sink node, when one node updated the other node does not update the path for whole multicast network but it updates the necessary nodes in the network.

First the network identifies the node which needs to be updated and after that the FTSP updates the shortest path for this identified node.

\subsection{Router}

Router is used to route the packet from source to destination in the network. It is consist of source node, destination node and 13 nodes from N1 to N13. The router stores information of a network in a database. In this database it stores attacker details like hacker which specify whether any attack is occurred in the network or not. The IP-address field shows the IP-address of the node on which attack is occurred. Node name tells the node number on which attack is occurred. The date field display the date and time of attack is occurred.

The node status details shows the detail information of node name, destination node, digital signature which are used for security and keep the information of whether any active or passive attack is occurred in the network or not. It also keep the node compromising attacker details like if there is any attack occurred then it shows yes otherwise it displays no.

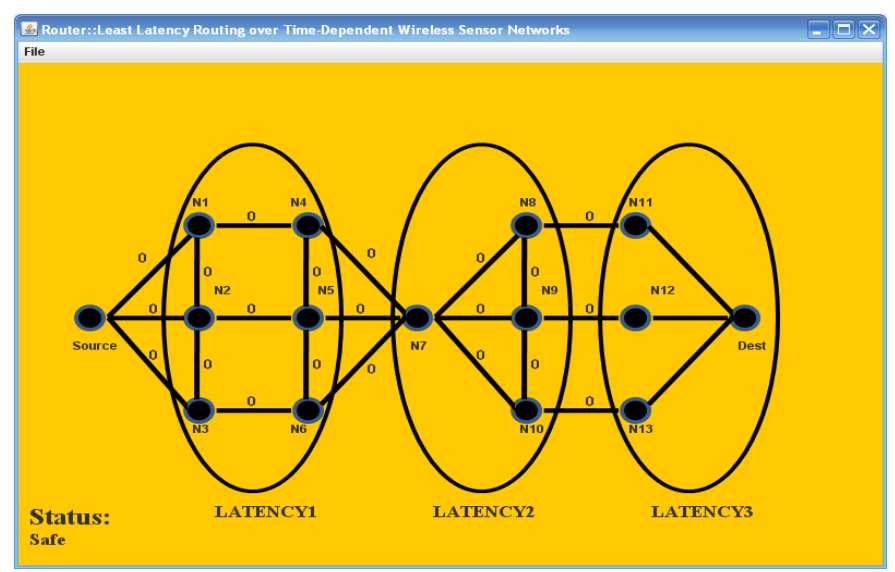

Fig. 1 Router with nodes from N1 to N13

\subsection{Sender}

Sender module has the browse button which selects the file from the system to forward to the destination. The initialize node button is then used to initialize the nodes in the network by accepting the IP-address of both the sender and receiver. After that the nodes will be initialized in the network.

The submit button is used to submit the data in the network and starts packet forwarding. When the packet is reach to the destination the popup window shows the message that the file is reach to the destination.

\subsection{Destination}

The destination window shows the file which is reached to the destination. And save this file into the receiver folder.

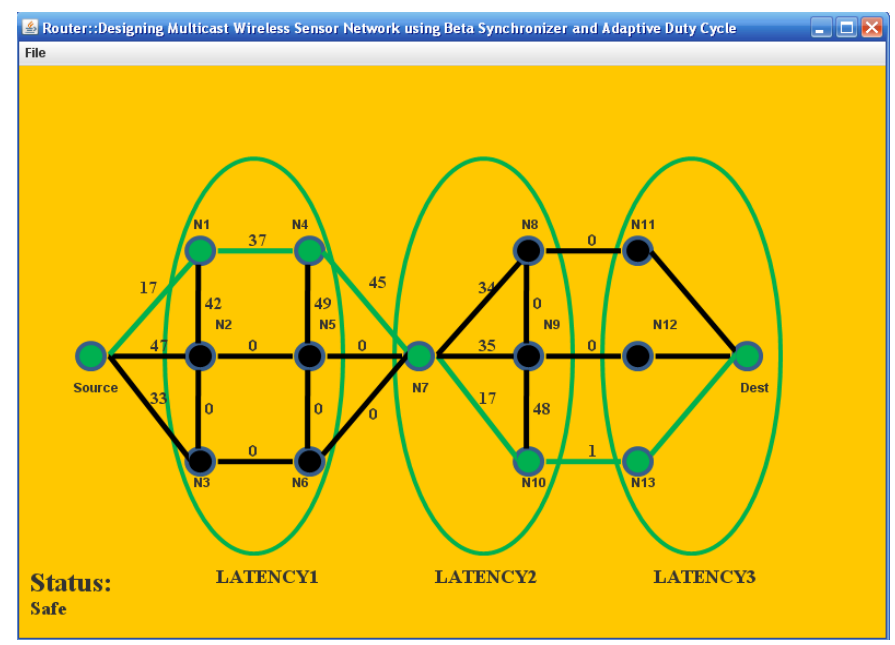

Fig. 2 Simulation of packet transmission with shortest path from source to destination 


\subsection{Passive Attacker}

The passive attacker is the attacker who can make attack on the node while communication is going on. When this attack is detected on the router, the router blocks that node and the sink hole is created.

\subsection{Active Attacker}

The active attacker is the known attacker who can make the attack while communication is going on. When this attack is detected on the router, the router blocks that node and the sink hole is created.

\subsection{Node Compromising Attack}

When the node compromising attack is occurred in the network, the network then chooses the other safe node for forwarding the packet to the destination.

\section{RESULTS}

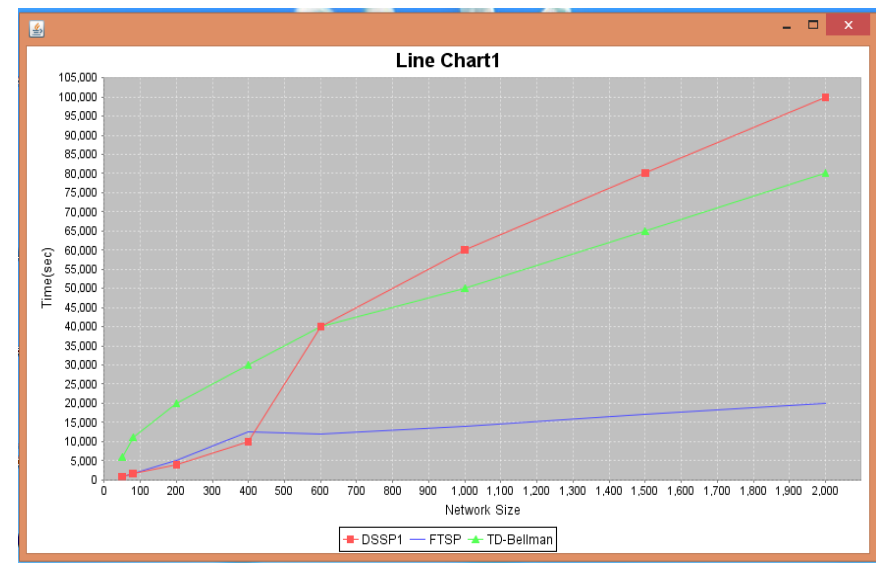

Fig. 3 FTSP takes minimum time comparing to TD-Bellman Ford and DSSP1.

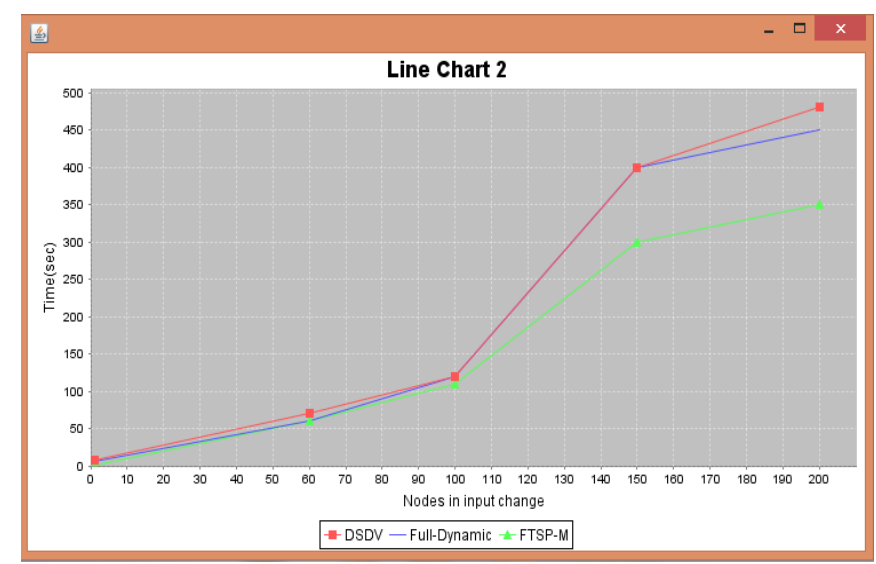

Fig. 4 When nodes change the input the FTSP-M takes less memory as compared to DSDV and Full Dynamic system.

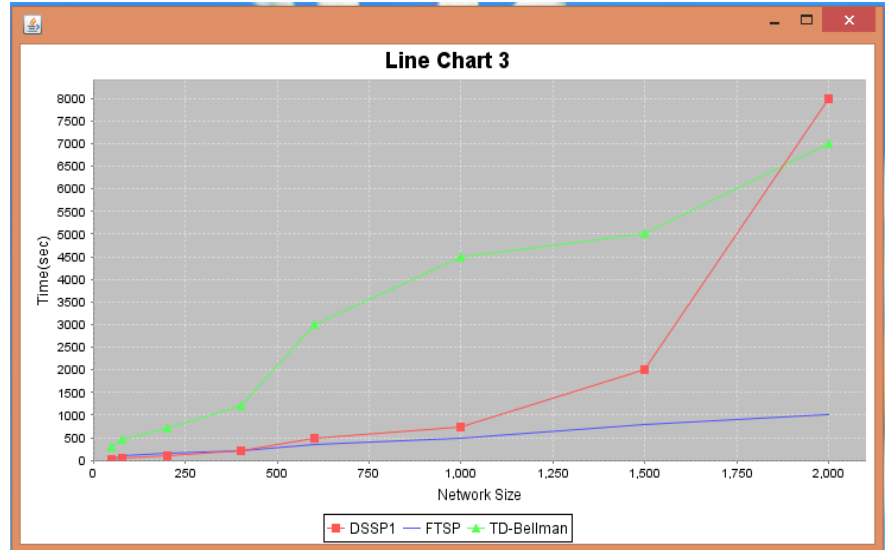

Fig. 5 when network size increases FTSP takes less average time to complete the process.

\section{CONCLUSIONS}

In this paper we have studied the actual simulation results which show that the FTSP algorithm with $\beta$ synchronizer decreases message, space and time complexity of the multicast WSN as compared to the traditional Bellman Ford algorithm. For decreasing the time, space and message complexity of a node we also studied the distributed shortest path routing problem in duty cycled wireless sensor network using $\beta$ synchronizer and adaptive duty cycle.

\section{REFERENCES}

[1] S. Lai and B. Ravindran, "Least-Latency Routing over Time Dependent Wireless Sensor Networks," in IEEE transaction 2013

[2] S. Lai and B. Ravindran, "On Distributed TimeDependent Shortest Paths over Duty-Cycled Wireless Sensor Networks," in IEEE INFOCOM, 2010

[3] B. Ding, J.X. Yu, and L. Qin, "Finding Time-Dependent Shortest Paths over Large Graphs," Proc. 11th Int'1 Conf. Extending Database Technology (EDBT '08), pp. 205-216, 2008

[4] L. Su, C. Liu, H. Song, and G. Cao, "Routing in Intermittently Connected Sensor Networks," in IEEE, 2008.

[5] Y. Gu, T. He, M. Lin, and J. Xu, "Spatiotemporal Delay Control for Low-Duty-Cycle Sensor Networks," in IEEE, 2009

[6] G.F. Italiano, "Distributed Algorithms for Updating Shortest Paths (Extended Abstract)," Proc. Fifth Int'l Workshop Distributed Algorithms 2000.

[7] S.Lai, B. Zhang, B. Ravindran, and H.Cho, "CQS-Pair: Cyclic Quorum System Pair for Wakeup Scheduling in Wireless Sensor Networks," 2008.

[8] P. Sommer and R. Wattenhofer, "Gradient Clock Synchronization in Wireless Sensor Networks," In ACM 2009. 
[9] Y.Sun, O. Gurewitz, and D.B. Johnson, "RI-MAC: A Receiver Initiated Asynchronous Duty Cycle MAC Protocol for Dynamic Traffic Loads in Wireless Sensor Networks," In ACM, 2008.

[10] W. Ye, J. Heidemann, and D. Estrin, "Medium Access Control With Coordinated Adaptive Sleeping for Wireless Sensor Networks," In IEEE/ACM Transaction 2004.

[11] T.V. Dam and K. Langendoen, "An Adaptive EnergyEfficient Mac Protocol for Wireless Sensor Networks," in ACM 2003.

[12] M. Zuniga and B. Krishnamachari, "Analyzing the Transitional Region in Low Power Wireless Links," in IEEE 2004

[13] C. Schurgers and M.B. Srivastava,"Energy Efficient Routing in Wireless Sensor Networks," 2001.

[14] A. Woo, T. Tong, and D. Culler, "Taming the Underlying Challenges of Reliable Multihop Routing in Sensor Networks," 2003.

[15] C.M. Vigorito, D. Ganesan, and A.G. Barto, "Adaptive Control of Duty Cycling in Energy-Harvesting Wireless Sensor Networks," 2007

[16] K.L. Cooke and E. Halsey, "The Shortest Route through a Network with Time-Dependent Internodal Transit Times," J. Math Analysis Application 1966

[17] A. Orda and R. Rom, "Distributed Shortest-Path Protocols for Time-Dependent Networks," Distributed Computing, 1996.

[18] C. Serafino, D. Gabriele, F. Daniele, and N. Umberto, "A Fully Dynamic Algorithm for Distributed Shortest Paths," Theoretical Computer Science, 2003

[19] S. Haldar, "An "All Pairs Shortest Paths" Distributed Algorithm Using 2n2 Messages," J. Algorithms, 1997

[20] K.V.S. Ramarao and S. Venkatesan, "On Finding and Updating Shortest Paths Distributively," J. Algorithms, 1992

[21] B. Awerbuch, "Complexity of Network Synchronization,” J. ACM, 1985 